\title{
Correspondence
}

\section{Calreticulin surface exposure is abrogated in cells lacking, chaperone-mediated autophagy-essential gene, LAMP2A}

\author{
AD Garg ${ }^{1}$, AM Dudek ${ }^{1}$ and P Agostinis ${ }^{*, 1}$
}

Cell Death and Disease (2013) 4, e826; doi:10.1038/cddis.2013.372; published online 3 October 2013

Subject Category: Experimental Medicine

\section{Dear Editor,}

Surface-exposed calreticulin (ecto-CRT) and secreted ATP have emerged as incontrovertible danger signals. ${ }^{1-3} \mathrm{~A}$ surge in studies describing the impact of these danger signals has also spurred a tremendous interest in the discovery of signaling pathways regulating their 'emission'. Recent research has shown that the pathways regulating the emission of these danger signals exhibit a certain degree of 'plasticity' and context-dependency. ${ }^{1-4}$ This applies mainly to the pathways regulating ecto-CRT emission and ATP secretion induced by mitoxantrone $(\mathrm{MTX})^{2}$ and hypericinbased photodynamic therapy (Hyp-PDT). ${ }^{3-5}$

Irrespective of the inducers though, molecular components regulating endoplasmic reticulum (ER)-stress and autophagy have been found to form the 'core' of pathways behind ecto-CRT and secreted ATP. ${ }^{1,3,5}$ While ER-stress tends to regulate these pathways positively, ${ }^{1,3}$ macroautophagy has recently emerged to play a more contextual role. ${ }^{1,6}$ While on one hand, macroautophagy was found to positively regulate MTX-induced ATP secretion, ${ }^{2}$ on the other we found that macroautophagy negatively regulates Hyp-PDT-induced ecto-CRT ${ }^{6}$ (without affecting ATP secretion ${ }^{6}$ ). This further increased our curiosity regarding the role of autophagy pathways behind ecto-CRT/secreted ATP. Interestingly, it was recently shown that the lysosomal protein LAMP1 can regulate MTX-induced ATP secretion. ${ }^{2}$ We have previously shown that oxidative stress generated by Hyp-PDT along with macroautophagy can stimulate chaperone-mediated autophagy (CMA), a process mediated by the lysosomal protein, LAMP2A ${ }^{7,8}$ Cells lacking LAMP2A were found to be extremely sensitized toward Hyp-PDT-induced apoptotic cell death, which is mediated by severe reactive oxygen species-induced ER stress. ${ }^{7}$ On the back of these exciting results, we became curious about the impact this CMA-essential gene can have on cell-surface exposure of CRT and secretion of ATP.

To this end, we tested the ability of LAMP2A ${ }^{+/+}$and LAMP2A $^{-1-}$ cells to emit secreted ATP/ecto-CRT following MTX/Hyp-PDT treatments. MTX and Hyp-PDT induced significant secretion of ATP in LAMP2A ${ }^{+1+}$ cells as compared with the untreated cells (Supplementary Figure S1A).
Similarly, the LAMP2A ${ }^{-1-}$ cells also retained their capacity to secrete ATP following MTX/Hyp-PDT treatment, to the same extent as the LAMP2A ${ }^{+1+}$ cells (Supplementary Figure S1A).

Next, we analyzed the induction of ecto-CRT and found both MTX and Hyp-PDT to be potent inducers of ectoCRT in the LAMP2A ${ }^{+/+}$cells (Supplementary Figure S1B), as expected. ${ }^{4,5}$ Interestingly, we found that the LAMP2A ${ }^{-1-}$ cells exhibited a strong inability to emit ecto-CRT, not only following MTX/Hyp-PDT treatments but also in basal conditions (Supplementary Figure S1B). This complete abrogation of ecto-CRT in the absence of LAMP2A indicated an 'innate' dysfunction of ecto-CRT trafficking pathway in these cells. It has been well established that cells lacking LAMP2A exhibit a compensatory rise in macroautophagy, under basal conditions, to counter the absence of CMA. ${ }^{7,8}$ We had recently observed that ablation of macroautophagy (via ATG5-siRNA) increased Hyp-PDT-induced ecto-CRT. ${ }^{6}$ Thus, we wondered whether we can rescue ecto-CRT emission in LAMP2A ${ }^{-1-}$ cells if we knockdown ATG5 (ATG5 ${ }^{\mathrm{KD}}$ ). ATG5 ${ }^{\mathrm{KD}}$ in LAMP2A ${ }^{+/+}$ cells (Supplementary Figure S1C, left) further increased the induction of ecto-CRT after treatment (as compared with LAMP2A $^{+/+}$cells transfected with Scr-siRNA) (Supplementary Figure S1C, right), especially after HypPDT, as expected. ${ }^{6}$ Contrary to expectations though, ATG $^{\mathrm{KD}}$ in LAMP2A ${ }^{-1-}$ cells $\left(\sim 50 \%{ }^{\mathrm{KD}}\right.$; Supplementary Figure S1C, left) failed to re-establish ecto-CRT, both under basal and treated conditions (Supplementary Figure S1C, right). These results indicate that possibly the absence of CMA in LAMP2A ${ }^{-1-}$ cells underlies their inability to emit ecto-CRT (rather than compensatory rise in ATG5-regulated macroautophagy) - a premise that needs to be comprehensively investigated.

In conclusion, we found that LAMP2A is dispensable for both MTX and Hyp-PDT-induced ATP secretion. However interestingly, we observed that the absence of LAMP2A abrogates ecto-CRT emission (in basal and MTX/Hyp-PDT treatment scenarios) - a dysfunction that cannot be rescued by siRNA-based knock-down of ATG5. In future, it would be imperative to investigate whether there is a direct link between ecto-CRT emission and CMA induction.

${ }^{1}$ Cell Death Research and Therapy Unit, Department for Cellular and Molecular Medicine, University of Leuven (KULeuven), Leuven, Belgium

${ }^{*}$ Corresponding author: P Agostinis, Cell Death Research and Therapy Unit, Department for Cellular and Molecular Medicine, Campus Gasthuisberg, O\&N1, Herestraat 49, Box 901, 3000 Leuven, Belgium. Tel: +32 16 345715; Fax: +32 16 345995; E-mail: patrizia.agostinis@med.kuleuven.be 


\section{Conflict of Interest}

The authors declare no conflict of interest.

Acknowledgements. This work was supported by grants from the Fund for Scientific Research Flanders (FWO-Vlaanderen; G.0661.09, G.0728.10 and G.0584.12N) and KU Leuven (GOA/11/009) to PA; ADG is a postdoctoral fellow supported by the BOF Postdoctoral Mandate (PDM) from KU Leuven (PDMK/12/146). This paper presents research results of the IAP7/32, funded by the Interuniversity Attraction Poles Programme, initiated by the Belgian State, Science Policy Office. We thank Dr. AM Cuervo (Albert Einstein College of Medicine, NY, USA) for the LAMP2A $^{+1+}$ and LAMP2A ${ }^{-1-}$ cells.

1. Garg AD et al. Cell Death Differ 2013; e-pub ahead of print 17 May 2013; doi:10.1038/cdd.2013.48.

2. Martins I et al. Cell Death Differ 2013; e-pub ahead of print 12 July 2013; doi:10.1038 cdd.2013.75.
3. Dudek AM et al. Cytokine Growth Factor Rev 2013; 24: 319-333.

4. Garg AD et al. Cancer Immunol Immunother 2012; 61: 215-221.

5. Garg AD et al. EMBO J 2012; 31: 1062-1079.

6. Garg AD et al. Autophagy 2013; 9: 1292-1307.

7. Dewaele M et al. J Cell Mol Med 2011; 15: 1402-1414.

8. Massey AC et al. Proc Natl Acad Sci USA 2006; 103: 5805-5810.

cc) (i) $(\Theta)$ Cell Death and Disease is an open-access journal published by Nature Publishing Group. This work is licensed under a Creative Commons Attribution-NonCommercialNoDerivs 3.0 Unported License. To view a copy of this license, visit http://creativecommons.org/licenses/by-nc-nd/3.0/

Supplementary Information accompanies this paper on Cell Death and Disease website (http://www.nature.com/cddis) 